\title{
Neutrophil chemotaxis and adhesion in preterm babies
}

\author{
R Carr, D Pumford, J M Davies
}

\begin{abstract}
To investigate the increased susceptibility to infection of very immature preterm neonates, neutrophil chemotaxis, Mac-1 adhesion receptor expression, and adherence to human umbilical vein endothelial cell monolayers (HUVE) were examined in neonates born at $\leq 32$ weeks' gestation.

Chemotaxis of neutrophils from well preterm neonates towards casein or zymosan activated serum (ZAS) was reduced (mean SE) being for casein 88.6 (3.8) $\mu \mathrm{m}$; ZAS $76 \cdot 2$ (4.3) $\mu \mathrm{m}$ compared with adults (casein 117.8 (1.4) $\mu \mathrm{m}$; ZAS $112.1(1.4) \mu \mathrm{m})$, but similar to term neonate neutrophils (casein 92.7 (4.5) $\mu \mathrm{m}$; ZAS 75.9 (3.1) $\mu \mathrm{m})$. Stimulated Mac-1 expression showed a similar pattern: reduced on preterm neutrophils compared with adults, but similar to term neonates. Preterm and term neonate neutrophils were both hyperadherent to HUVE when unstimulated, but showed an equally impaired ability to increase adhesion following stimulation. Casein stimulated chemotaxis and stimulated Mac-1 expression 'matured' towards adult levels of performance four weeks after preterm birth.
\end{abstract}

The increased incidence of sepsis in immature preterm infants is not due to greater defects of neutrophil migration.

The incidence of severe neonatal sepsis is greatly increased in very immature preterm neonates born weighing less than 1500 g. $^{12}$ The immune system is still immature at the time of fullterm birth and the deficiencies in humoural and cellular immunity are even more appreciable in the very immature preterm infant. ${ }^{3}$

Neutrophil polymorphonuclear leucocytes provide the major defence against bacterial invasion. The neutrophil dysfunction of term infants has been explored in numerous studies $^{4}$ but there has been little work on the functional capacity of neutrophils from very immature preterm infants, or the changes that occur after preterm birth.

Neutrophils respond to the stimulus of bacterial invasion by upregulating adhesion receptors on the cell surface, increasing adherence to the adjacent capillary endothelium, and migrating from the circulation to localise at the site of infection. The most important neutrophil adhesion receptor mediating stimulated adhesion and chemotaxis is the integrin
Mac-1, defined by monoclonal antibodies (MoAb) to CD11b/CD18.5

Impaired neutrophil chemotaxis is a consistent finding in healthy term neonates. ${ }^{6-12}$ This has been shown to be associated with impaired adherence to artificial surfaces ${ }^{9-14}$ and a reduced ability to upregulate Mac-1 after cell stimulation. ${ }^{15}$ Two previous studies of neonate neutrophil chemotaxis have included preterm infants, ${ }^{11} 16$ but no previous study has investigated chemotaxis, Mac-1 expression, and adhesion in the group of immature preterm infants born before 32 weeks' gestation who are at the greatest risk of bacterial infection.

To examine further the increased susceptibility of preterm neonates to bacterial sepsis, we have measured chemotaxis and Mac-1 expression on neutrophils from well preterm infants born before 32 weeks' gestation and examined the changes that occur during early postnatal development. In addition, we have measured adhesion to endothelial monolayers as a more representative model of normal physiology than the reported adhesion assays using artificial surfaces.

\section{Patients and methods \\ STUDY POPULATION}

Twenty three 'well' preterm infants were studied soon after birth and at intervals during the first weeks of life. The median gestational age was 27 weeks (range 24-32) and median birth weight $1060 \mathrm{~g}$ (664-1952). Neonates were defined as 'well' if they had no evidence of infection and did not require mechanical ventilation for pulmonary insufficiency.

For analysis the infants were divided into groups: group 1, preterm neonates studied soon after birth at a median postnatal age of 1 day (range 1-4), $n=22$; group 2, preterm infants studied for a second time on median day 10 (5-21), $n=23$; group 3, preterm infants studied on median day $56(28-70), n=10$. For comparison, 28 healthy term neonates (group 4) were studied at the time of delivery by elective caesarean section, median gestational age $38(38-40)$ weeks. All infants were studied in parallel with healthy adult controls (group 5), $\mathrm{n}=80$.

The study was approved by and all blood sampling was in accordance with the Liverpool Maternity Hospital ethics committee guidelines.

ISOLATION OF NEUTROPHILS

Polymorphonuclear leucocytes from preterm neonates were isolated from umbilical arterial 
catheter or peripheral venous samples; from term neonates using cord blood collected at birth; and from adults by venepuncture. Blood was collected into heparin $10 \mathrm{U} / \mathrm{ml}$ and neutrophils separated on a sodium metrizoate/ dextran gradient (Polyprep, Nycomed). Residual red cells were removed by hypotonic lysis and resuspended in Roswell Park Memorial Institute (RPMI) medium with calcium and magnesium (Flow Laboratories), the cell suspensions and all reagents being kept on ice. The purity of the neutrophil preparations was assessed by visual inspection of cytospin preparations. Viability, by trypan blue exclusion, was $>95 \%$ in all cases.

\section{PREPARATION OF REAGENTS}

Stock solutions of f-Met-Leu-Phe (FMLP) (Sigma) were prepared in phosphate buffered saline (PBS). Casein (Sigma) was prepared as a $5 \%$ stock solution in PBS and before use diluted 1:10 in Hanks' BSS solution (Sigma). Zymosan activated serum (ZAS) was prepared by incubating zymosan (Sigma) with pooled fresh $\mathrm{AB}$ serum for 45 minutes at $37^{\circ} \mathrm{C}$ followed by 30 minutes at $56^{\circ} \mathrm{C}$. Rose Bengal (Sigma) was prepared as a $0.25 \%$ solution in PBS; glutaraldehyde $(\mathrm{BDH})$ as a $2 \%$ solution in PBS

\section{OUANTITATION OF MAC-1 RECEPTORS}

Mac- $1 \alpha$ chain (CD11b) was detected using MoAb Leu15 (Becton Dickinson); measurements were made on unstimulated neutrophils and after stimulation with $10^{-8} \mathrm{M}$ FMLP for 15 minutes at $37^{\circ} \mathrm{C}$. Cells were labelled for indirect immunofluorescence using saturating concentrations of MoAb and FITC goat antimouse IgG (Becton Dickinson). Surface stained cells were fixed in $1 \%$ paraformaldehyde and analysed on a Becton Dickinson FACS Analyser I. Forward and $90^{\circ}$ light scatter were used to identify and isolate the neutrophil population for analysis. Fluorescence intensity distribution of 5000 cells was recorded on a three decade logarithmic scale and the mean fluorescence channel for each cell population calculated.

\section{CHEMOTAXIS}

Chemotaxis through a $3 \mu \mathrm{m}$ pore size Millipore filter was measured in a modified Boyden chamber. Neutrophils, resuspended in Hanks' BSS with $1 \%$ bovine serum albumin were placed in the upper chamber and migrated towards casein or ZAS placed in the lower chamber. After 90 minutes' incubation the leading front distance was measured on a calibrated microscope.

\section{ADHESION TO HUMAN UMBILICAL VEIN}

ENDOTHELIAL CELLS

Conventional adhesion assays require large numbers of neutrophils. To allow adhesion to human umbilical vein endothelial cells (HUVE) to be assayed within the limits of the small cell numbers available from preterm infants, an assay system was developed using Rose Bengal staining.

HUVE cells were grown to a confluent monolayer in flat bottomed microtitre plate wells. Neutrophil suspensions in RPMI were adjusted to $5 \times 10^{6} \mathrm{cells} / \mathrm{ml}$, warmed to $37^{\circ} \mathrm{C}$, and $100 \mu \mathrm{l}$ per well layered onto the HUVE with (stimulated) or without (unstimulated) $10^{-6}$ FMLP (final concentration). Each test suspension was added to four identical wells. To allow complete settling of the neutrophils onto the HUVE, the plate was incubated undisturbed for 15 minutes at $37^{\circ} \mathrm{C}$ before $100 \mu \mathrm{l}$ of $2 \%$ glutaraldehyde solution was gently layered into each well to fix adherent neutrophils. Non-adherent neutrophils were removed by two washes with RPMI pipetted into the wells and removed by aspiration. Then $100 \mu \mathrm{l}$ of $0.25 \%$ Rose Bengal was added to each well and incubated for 10 minutes after which any stain not absorbed into the cells was removed by four vigorous washes with PBS. To release absorbed stain from the cells into solution, ethanol (diluted $1: 1$ in PBS) was added to each well and incubated for 1.5 hours.

Optical density was measured at $570 \mathrm{~nm}$ in a Titertek Multiscan Plus microtitre plate reader, and the mean optical density of each set of quadruplicate wells taken. To quantify the number of adherent neutrophils the optical density of wells containing HUVE alone (mean of four) was subtracted from the optical density of each quadruplicate set of test wells.

Previous validation experiments had shown a linear relationship between the number of adherent neutrophils in a well and the optical density resulting from Rose Bengal staining.

\section{Calculation of an adhesion index}

This assay does not allow quantitation of the absolute number of adherent cells, and therefore results were related to the adherence of FMLP stimulated adult control cells (standard control), which was taken to represent an adhesion index of $100 \%$. As the absolute number of adherent cells is related to the number of cells added to each well, all test and control neutrophil suspensions were adjusted to the same cell concentration. As an additional precaution, each test cell suspension was recounted at the time of dispensing into wells; these counts were used to make adjustment for any minor variation between cell number added to test wells and those added to control wells. The following formulas were used.

Adjusted optical density (OD)=

$$
\begin{aligned}
& \begin{array}{l}
\text { Measured OD adherent cells } \\
\text { (that is, OD test well-OD HUVE) }
\end{array} \\
& \text { then: } \\
& \text { Adhesion index (\%) }=\frac{\text { standard control cell count }}{\text { test cell count }} \\
& \text { OD standard control }
\end{aligned}
$$

\section{STATISTICAL ANALYSIS}

Results are described by mean and standard error (SE). Study groups are compared by a two sample $t$ test. Changes occurring within a single study population are additionally described by $95 \%$ confidence intervals $(95 \% \mathrm{CI})$. 
Analysis of neonatal neutrophil adhesion to HUVE monolayers is compared with the simultaneously tested adult controls by a paired $t$ test because of the variation in performance between HUVE batches.

\section{Results}

Chemotaxis, Mac-1 (CD11b/CD18), and adherence to HUVE monolayers were measured sequentially in 23 clinically well preterm neonates born between 24 and 32 weeks' gestation. For comparison, 28 term neonates were studied at the time of birth.

\section{CHEMOTAXIS}

Random cell movement (chemokinesis) in all preterm neonate groups and in term infants was similar to adult controls (table). There was no statistically significant difference between groups.

Mac-1 expression and chemotaxis by study group. Values shown as mean (SE)

\begin{tabular}{|c|c|c|c|c|c|}
\hline \multirow{2}{*}{$\begin{array}{l}\text { Study group } \\
\text { (postnatal age) }\end{array}$} & \multicolumn{2}{|c|}{ Fluorescence } & \multirow[t]{2}{*}{ Chemokinesis ( $\mu \mathrm{m}$ ) } & \multicolumn{2}{|c|}{ Chemotaxis ( $\mu \mathrm{m}$ ) } \\
\hline & $\overline{M a c-1 R}$ & $M a c-1 S$ & & Casein & $Z A S$ \\
\hline $\begin{array}{l}\text { (1) Well preterm } \\
\text { (days 1-4) }\end{array}$ & $15 \cdot 4(1 \cdot 2)$ & $72 \cdot 5(4 \cdot 2)$ & $42.9(2 \cdot 3)$ & $88.6(3.8)$ & $76 \cdot 2(4 \cdot 3)$ \\
\hline $\begin{array}{l}\text { (2) Well preterm } \\
\text { (days } 5-21 \text { ) }\end{array}$ & $15 \cdot 6(0.8)$ & $75 \cdot 2(2 \cdot 4)$ & $42 \cdot 3(3 \cdot 1)$ & $91 \cdot 7(4 \cdot 4)$ & $81 \cdot 2(5 \cdot 4)$ \\
\hline $\begin{array}{l}\text { (3) Well preterm } \\
\text { (days 28-70) }\end{array}$ & $13.9(1.9)$ & $110 \cdot 2(3 \cdot 8)$ & $41 \cdot 3(2 \cdot 0)$ & $104.0(4 \cdot 4)$ & $80 \cdot 6(7 \cdot 0)$ \\
\hline $\begin{array}{l}\text { (4) Term neonates } \\
\text { (day 1) }\end{array}$ & $18 \cdot 5(1.4)$ & $85.7(3.9)$ & $41.6(2 \cdot 6)$ & $92 \cdot 7(4 \cdot 5)$ & $75 \cdot 9(3 \cdot 1)$ \\
\hline (5) Adult & $11.9(0.3)$ & $133.9(2.3)$ & $42.4(1 \cdot 2)$ & $117.8(1.4)$ & $112 \cdot 1(1 \cdot 4)$ \\
\hline
\end{tabular}

Mac-1R: Mac-1 expression on unstimulated neutrophils.

Mac-1S: Mac-1 expression on neutrophils after stimulation with FMLP.
Directed migration (chemotaxis) was assessed in response to casein (fig 1, table) and ZAS (table). In response to either stimulus, the chemotaxis of neutrophils from well preterm neonates did not differ significantly from term neonates. However, leading front migration of both preterm and term neonate neutrophils was significantly reduced compared with simultaneously tested adult cells: $\mathrm{p}<0.0001$ for group $1 v$ group 5 , and for group $4 v$ group 5.

For preterm neonates tested at birth there was no correlation between chemotaxis and gestational age (casein $r=-0.234$; ZAS $r=$ $-0.105)$, or between chemotaxis and birth weight (casein $r=-0 \cdot 142$; ZAS $r=-0 \cdot 216$ ).

The preterm infants were studied again between the fifth and 21 st postnatal day (group 2); all were well at the time of testing. Chemotactic responses were similar to those at birth (table). Postnatal maturation, represented by an increase in chemotactic response to casein, was observed in healthy infants after the fourth week following preterm birth (group 3) (fig 1, table): mean increase (group $1 v$ group 3 ) $=15.4 \mu \mathrm{m} ; 95 \%$ CI $3 \cdot 1$ to $27 \cdot 8$; $\mathrm{p}=0 \cdot 02$. But cell migration remained impaired in comparison to adult neutrophils, $\mathrm{p}=0.02$. Migration towards ZAS, however, did not increase significantly over a similar postnatal period: mean increase (group $1 v$ group 3)= $4.4 \mu \mathrm{m}$ (95\% CI-13.9 to 22.7$)$. The difference in chemotaxis towards ZAS and casein in the postnatal period is unexplained, but may relate to differences in the clinical condition of the infants studied with each agent.

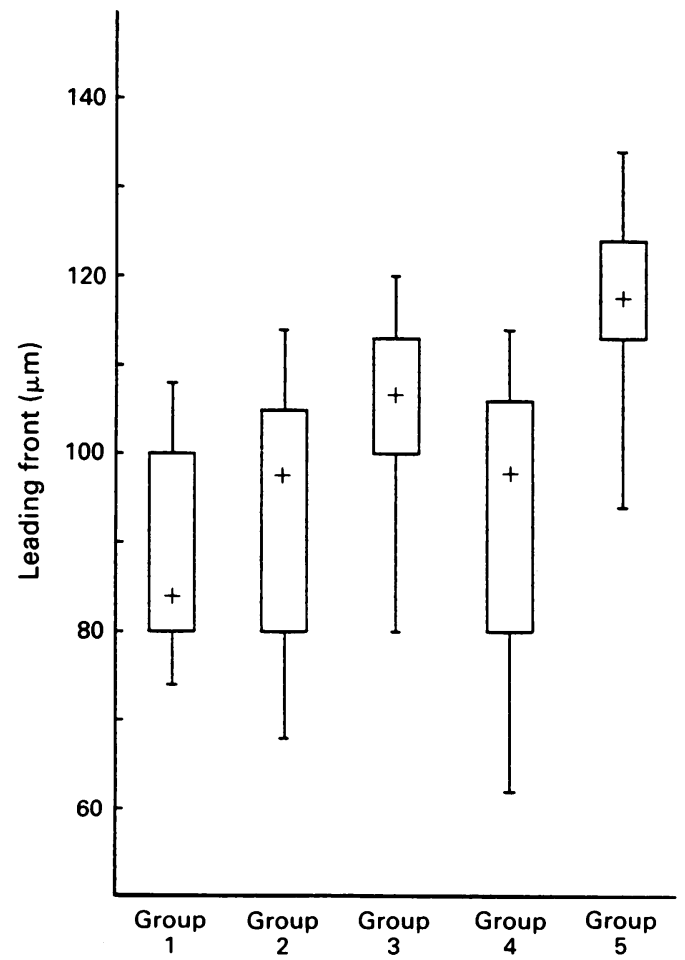

Figure 1 'Box plot', showing median and quartiles, of neutrophil chemotaxis (leading front distance) towards casein by study group. Group $1=$ well preterm days 1-4, group $2=$ well preterm days $5-21$, group $3=$ well preterm days 28-70, group 4=term neonates (day 1), group 5=adults.

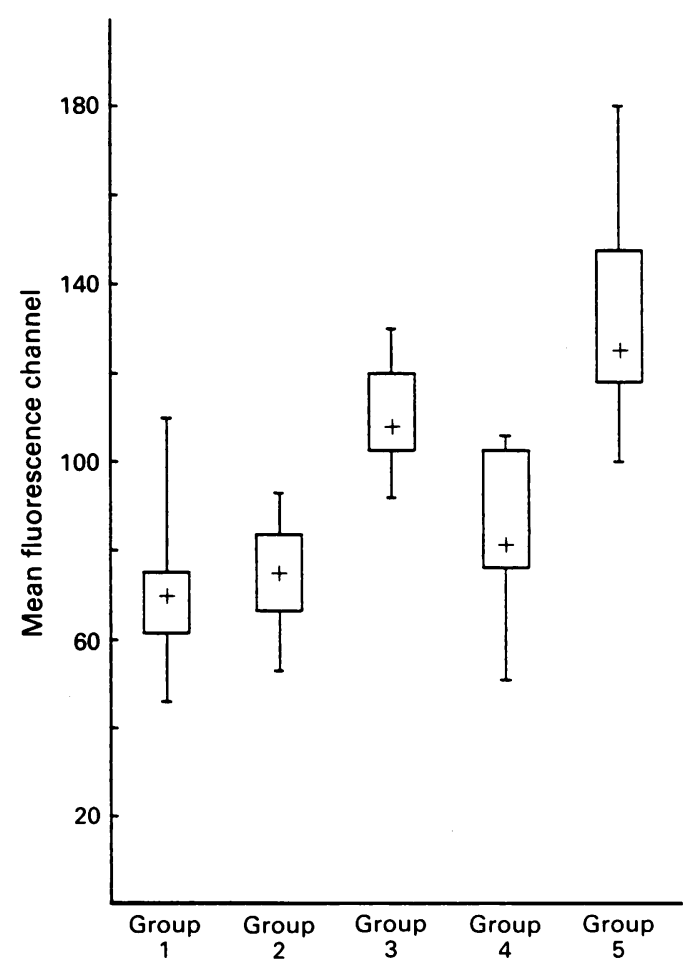

Figure 2 'Box plot' of Mac-1 $\alpha$ expression on FMLP stimulated neutrophils by study group. Group $1=$ well preterm days 1-4, group $2=$ well preterm days 5-21, group $3=$ well preterm days $28-70$, group $4=$ term neonates (day 1), group 5=adults. 
MAC-1

The pattern of expression of Mac- $1 \alpha$ chain (CD11b) on the neutrophil cell membrane after FMLP stimulation had some similarity with the observed chemotactic responses (fig 2). FMLP stimulated Mac-1 $\alpha$ expression was significantly impaired in both healthy preterm neonates, and term neonates, compared with adult controls: $p<0.0001$ for group $1 v$ group 5, and group $4 v$ group 5 (table). Stimulated Mac-1 $\alpha$ expression on preterm neutrophils was slightly less than on term neonate cells: $p=0 \cdot 03$.

After preterm birth, the ability to upregulate Mac-1 $\alpha$ improved in line with casein stimulated chemotaxis. After the fourth postnatal week (group 3) stimulated Mac-1 $\alpha$ expression had significantly increased by a mean of 37.7 (95\% CI 25.9 to 49.5$)$; $\mathrm{p}<0.0001$. However while Mac- $1 \alpha$ expression by neutrophils from these older infants overlapped the adult range, the mean Mac-1 $\alpha$ expression remained below the adult group mean (fig 2 , table).

\section{ADHESION TO HUVE}

The adherence to unstimulated HUVE monolayers by unstimulated and stimulated neutrophils was examined in 16 term and seven well preterm neonates soon after birth (fig 3). Unstimulated neutrophils from both term and preterm neonates were hyperadherent compared with simultaneously tested adult control cells. The mean (SE) adhesion index of unstimulated term neonate neutrophils was $83.9(6.5) \% v$ adult neutrophils $56.6(4.7) \%$, $\mathrm{p}=0.01$; unstimulated preterm neutrophils, $69 \cdot 7(6.0) \% v$ adults $46.9(6 \cdot 0) \%, p=0.04$. There was no significant difference between the adherence of unstimulated cells from term and preterm infants.

After FMLP stimulation, term neonate neutrophil adherence increased by a mean adhesion index of $25.6 \%(95 \%$ CI $16 \cdot 1$ to $35 \cdot 1)$, and preterm neonate neutrophils by a mean of $20.1 \%$ (95\% CI 6.8 to 33.5$)$. Simultaneously tested adult controls increased

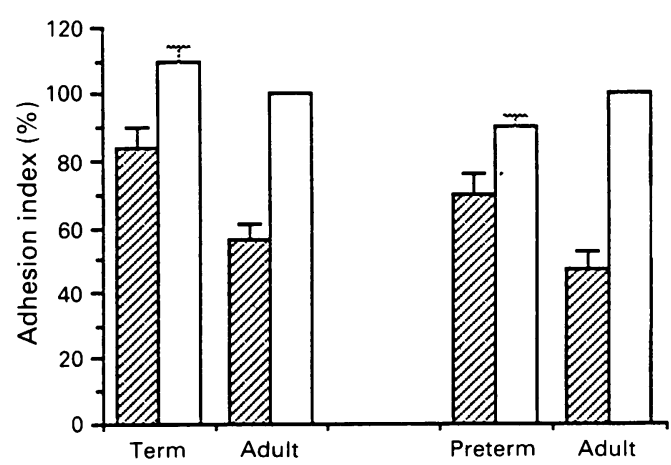

Figure 3 Bar chart showing mean (SE) adhesion index (\%) to HUVE monolayers. The open bars represent FMLP stimulated neutrophil adhesion; cross hatched bars represent unstimulated adhesion. The 16 term infants are paired graphically with the simultaneously tested adult controls. The seven well preterm infants are similarly paired with their own adult controls. The bars representing stimulated adult neutrophil adhesion have no error bar as the adhesion index is, by definition, $100 \%$ (see methods). adherence after FMLP stimulation by $43.4 \%$ (95\% CI 33.4 to $53 \cdot 4)$, and $53.1 \%$ (95\% CI $38 \cdot 5$ to $67 \cdot 8$ ), respectively. Thus neutrophils from both term and preterm neonates exhibited significantly impaired upregulation of adherence in response to FMLP when compared with simultaneously tested adult cells, $p=0.01$ and $p=0.002$ respectively.

\section{Discussion}

This study has examined three aspects of neutrophil migration in healthy and stressed preterm neonates born between 24 and 32 weeks' gestation: expression of the Mac-1 (CD11b) component of the integrin adhesion complex (CD11/CD18), adhesion to a physiological substrate (HUVE monolayers), and chemotaxis.

Neutrophils from healthy preterm neonates uniformly displayed chemotactic responses and stimulated Mac-1 upregulation that were truncated compared with normal adults but which were no different from the responses of healthy fullterm neonates. After preterm birth casein stimulated chemotaxis and FMLP stimulated Mac-1 upregulation increased to approach adult levels after the fourth postnatal week.

These data support and extend the observations of others that neutrophil chemotactic ability is not related to gestational age. Krause et al studied four healthy newborn infants $<1500 \mathrm{~g}^{11}$ and Bektas et al 13 newborn infants of unspecified clinical status born between 28 and 36 weeks. ${ }^{16}$ Both found that the preterm and term neonate neutrophils migrated equally towards ZAS.

The postnatal maturation of chemotactic ability after very premature birth has not previously been examined. Sacchi et al studied five infants born before 34 weeks and found delayed maturation of chemotaxis compared with four infants born between 34 and 36 weeks. ${ }^{17}$ It was suggested that the delayed maturation may have been secondary to the increased number of infections in the more immature group. The same study found that a group of 10 term infants had established normal neutrophil chemotaxis by the time they and regained their birth weight. A more recently longitudinal study of healthy term neonates showed that normal adult levels of chemotaxis towards ZAS were established between 10 and 32 days after birth. ${ }^{12}$

In the present study of well preterm neonates born between 24 an 32 weeks' gestation, the postnatal increase in casein stimulated neutrophil migration was only slightly delayed in comparison to that reported for term infants. After the fourth week following preterm birth the chemotactic response of seven out of nine infants tested overlapped the adult range. Thus even in immature preterm neonates there is a rapid postnatal maturation of both chemotaxis and the ability to upregulate Mac-1, while these aspects of neutrophil function remain depressed and at a constant level throughout normal uterine development to term. 
Likewise, neutrophils from preterm and term neonates behaved similarly in the adhesion assay, with a reduced ability to upregulate adherence compared with normal adult cells. It was not possible to do sequential postnatal studies of adherence in the preterm infants on account of the low number of circulating neutrophils in well infants after the first 10 postnatal days. ${ }^{18}$

Previous studies of neonate neutrophil adherence have examined adhesion to artificial surfaces, with variable results. ${ }^{9-1416}$ Only one study, by Anderson et al, has examined adherence to endothelium. ${ }^{19}$ This study, which included term neonates only, agreed that their neutrophils had impaired upregulation of adherence when stimulated. However, in contrast to our finding that unstimulated neonate cells were hyperadherent, this published study reported reduced adherence to HUVE by unstimulated neonate cells. The difference may stem from differences in the adhesion assay techniques. In our assay adhesion to HUVE was measured at a time when adherence was maximal ${ }^{20}$ by fixing the cell interactions at 15 minutes with glutaraldehyde. The longer neutrophil/HUVE interaction used by Anderson's group may mean that adhesion was measured after the hyperadherent phase had passed. 1921

The similar functional responses of neutrophils from term infants and preterm neonates born before 32 weeks' gestation suggests that the increased incidence of severe infection in immature preterm newborn infants is not related to differences in the ability of their neutrophils to adhere and migrate. The similarity between term and preterm neonate neutrophils suggests a state of arrested functional 'maturation' from at least 25 weeks' gestation in the developing fetus. The point in ontogeny at which neutrophils of the developing fetus reach this functional steady state, the mechanisms which maintain fetal neutrophil function in developmental limbo, and the changes that occur at birth to trigger the process of maturation are unknown.

We thank Professor R W I Cooke and Dr A M Weindling for permission to study infants under their care at the Liverpool Maternity Hospital. Thanks also go to Joyce Billington fo additional technical assistance.
The study received financial support from Mersey Regional Health Authority.

1 Hensey O, Hart CA, Cooke RWI. Serious infection in a neonatal intensive care unit: a two year survey. $\mathcal{f} H y g$ (Camb) 1985;95:289-97.

2 de Louvois J. Septicaemia and meningitis in the newborn. In: de Louvois J, Harvey D, eds. Infection in the newborn. Perinatal practice. 1990;6:107-15.

3 Whitelaw A, Parkin J. Development of immunity. $\mathrm{Br} \mathrm{Med}$ Bull 1988;44:1037-51.

4 Hill HR. Biochemical, structural and functional abnormalities of polymorphonuclear leukocytes in the neonate. Pediatr Res 1987;22:375-82.

5 Anderson DC, Miller LJ, Schmalstieg FC, Rothlein R, Springer TA. Contributions of the Mac-1 glycoprotein family to adherence-dependent granulocyte functions: structure-function assessments employing subunit-spestructure-function assessments employing subunit-spe-

6 Miller ME. Chemotacic function in the human neonate: humoral and cellular aspects. Pediatr Res 1971;5: humoral

7 Pahwa S, Pahwa R, Grimes E. Cellular and humoral components of monocyte and neutrophil chemotaxis in cord blood. Pediatr Res 1977;11:677-80.

8 Klein RB, Fischer TJ, Gard SE. Decreased mononuclear and polymorphonuclear chemotaxis in newborns. Pediatrics 1977;60:467

9 Anderson DC, Hughes BJ, Smith CW. Abnormal mobility of neonatal polymorphonuclear leukocytes: relationship to impaired redistribution of surface adhesion sites by chemotactic factor or colchicine. 7 Clin Invest 1981;68: 863-74.

10 Boner A, Zeligs BJ, Bellanti JA. Chemotactic responses of various differentiational stages of neutrophils from human cord and adult blood. Infect Immun 1982; 35: 921-8.

11 Krause PJ, Herson VC, Boutin-Lebowitz J, et al. Polymorphonuclear leukocyte adherence and chemotaxis in stressed and healthy neonates. Pediatr Res 1986;20: 296-300.

12 Eisenfeld L, Krause PJ, Herson V, et al. Longitudinal study of neutrophil adherence and motility. $\mathcal{F}$ Pediatr 1990 117:926-9.

13 Krause PJ, Maderazo EG, Scroggs M. Abnormalities of neutrophil adherence in newborns. Pediatrics 1982;69: 184-7.

14 Harris MC, Levitt J, Douglas SD, Gerdes JS, Polin RA. Effect of fibronectin on adherence of neutrophils from newborn infants. $\mathcal{f}$ Clin Microbiol 1985;21:243-6.

15 Anderson DC, Becker Freeman KL, Heerdt B, Hughes BJ, Jack RM, Smith CW. Abnormal stimulated adherence of neonatal granulocytes: impaired induction of surface Mac-1 by chemotactic factors or secretagogues. Blood 1987;70:740-50.

16 Bektas S, Goetze B, Speer CP. Decreased adherence, chemotaxis and phagocytic activities of neutrophils from preterm neonates. Acta Paediatr Scand 1990;79:1031-8.

17 Sacchi F, Rondini G, Mingrat G, et al. Different maturation of neutrophil chemotaxis in term and preterm newborn infants. $\mathcal{F}$ Pediatr 1982; 101: $273-4$

18 McIntosh N, Kempson C, Tyler RM. Blood counts in extremely low birthweight infants. Arch Dis Child 1988, 63: 74-6.

19 Anderson DC, Rothlein R, Marlin SD, Krater SS, Smith $C W$. Impaired transendothelial migration by neonatal neutrophils: abnormalities of Mac-1 (CD1 1b/CD18)dependent adherence reactions. Blood 1990;76:2613-21.

20 Lo SK Van Seventer GA Levin SM, Wright SD. Two leukocyte receptors (CD112/CD18 and CD11b/CD18) leukocyte receptors (CD11a/CD18 and CD11b/CD18) mediate transient adhesion to endothelium by bin

21 Smith CW, Hollers JC, Patrick RA, Hassett C. Motility and adhesiveness in human neutrophils: effects of chemotactic factors. $\mathcal{f}$ Clin Invest 1979;63:221-9. 